\title{
Design Failure Mode and Effects Analysis (DFMEA) for Automotive Safety Systems
}

\author{
Dorin-Ion DUMITRASCU \\ Transilvania University of Brasov, Romania, d.dumitrascu@unitbv.ro \\ Adela-Eliza DUMITRASCU \\ Transilvania University of Brasov, Romania, dumitrascu a@unitbv.ro
}

\begin{abstract}
In this paper is presented an effective application of Failure Modes and Effects Analysis (DFMEA) in product design stages specific to vehicle safety systems. The scope of DFMEA is to eliminate as early as possible the design faults that could lead to the defective products. The potential failure modes, its causes, and effects are identified and severity, likelihood and detection are quantified in order to provide the corrective actions before first production run occurs. The results analysis for optimized stage indicate that all the analysed characteristics have a priorityrisk level (RPN) located in the medium and low risk areas, which means an improvement of the design process performance.
\end{abstract}

\section{Keywords}

failure modes and effects analysis, vehicle safety systems, risk priority-number (RPN), design improvement

\section{Introduction}

Analysis of failure modes and their effects (FMEA) is a method of analysing the potential defects of a product or process in order to develop a set of measures aimed to prevent them and to increase the quality level of products, work processes and production environments [1, 2, 3].

DFMEA is a methodical approach used for identifying potential risks introduced in a new or changed design of a product or service. The Design FMEA initially identifies design functions, failure modes and their effects on the customer with corresponding severity ranking / danger of the effect. Then, causes and their mechanisms of the failure mode are identified [4].

DFMEA is a methodology to identify and prevent the potential failure modes of products that are related to their design, in order to validate the established design parameters for a specific functional performance level, at system, subsystem or component level. It aids in the objective evaluation of design requirements and design alternatives $[5,6,7]$. It provides additional information to aid in the planning of efficient design testing. In order to eliminate or mitigate the effects, DFMEA plays an important role by selecting the optimal design variant and develop a documentary base to support future designs in order to reduce the risks which is associated with the faulty products that reach to the customers [7].

By implementing the DFMEA method, the potential risk of defects from the product design stage is reduced. In this sense, DFMEA will ensure the reduction of the costs of non-quality in all the specific stages of the processes of:

- Design: through a better knowledge of the clients' requirements.

- Supply: avoiding problems generated by improper selection of suppliers and subsequently, reducing customer complaints.

- Production: prevention of potential critical points.

\section{Safety Systems for Cars}

Occupant safety systems must provide automatic protection in the case of any type of collision or rollover of a vehicle. In these cases, the seat belt and airbag represent passive restraint systems.

In accordance with the European safety regulations, seat belts are mandatory for all seats in the car and airbags are mandatory for the driver and front passenger $[8,9,10,11]$.

By wearing the seat belt we ensure that: 
- we reduce the risk of injury or death by over $40 \%$;

- we distribute the forces on bigger and stronger parts of the human body;

- the movement allowed by the seat belt during the accident, does not apply instantaneously the force on the body so the passenger gradually decelerates (the seat belt absorbs a level of energy);

- without wearing the seat belt, these occupants put in danger both, their lives and those of other car passengers.

Cars equipped with airbags manage to:

- reduce the risk of injury or death by up to $20-35 \%$;

- airbags like seat belts spread the impact energy over a larger body surface;

- protects the head, chest and knees against the side windows, the dashboard and other strong parts of the passenger compartment.

- airbags supplement the seat belt and do not replace it, but airbags also protects occupants who are not wearing seat belts.

Due to the combined use of the seat belt and airbag, the serious injuries that occur in the case of frontal collisions can be reduced by $65 \%$ at the chest level and up to $75 \%$ for the head.

The seat belt provided with a pretensioner and an airbag, used together, form at present time the most effective restraint system in the case of a serious accident. At speeds exceeding $40 \mathrm{~km} / \mathrm{h}$ the seat belt alone does not provide sufficient protection. Research into the consequences of accidents has established that in $68 \%$ of cases, an airbag ensures a significant improvement in passenger's safety.

The current legislation obliges the car manufacturers to provide the cars with mandatory safety systems and, as the case may be, to obtain more stars in the Euro NCAP tests, the manufacturers equip the cars with optional safety systems. In order to sell the vehicles, manufacturers must approve the cars with the required safety systems.

\section{Case Study}

The case study refers to the application of the DFMEA analysis specific to the seat belt locking system strap. Due to the large volume of data and the fact that changes in the concept occur very often in the design process, the design method is realized using computer-aided design software (e.g. Catia V5). These design software offers the possibility of relatively easy modification of already existing 3D models, the analysis of geometry defects and interference between different components, the analysis of the inclination of the faces of the housings in order to extract the parts from the injection moulds, creating drawings based on 3D models, etc.

The following criteria are defined:

- Critical criteria - dimensions and functions that are $100 \%$ followed and controlled;

- Significant criteria - dimensions and functions that are followed and controlled with certain established frequencies.

The assessment methodology consists of assessment of severity of the failure, likelihood of occurrence of failure and likelihood of detection. The failure is determined for each failure mode.

The potential causes of failure were identified, which were quantified from the point of view of occurrence, as well as the control methods for each specified cause, where the detection scores were given, resulting in the risk score for the initial stage of the design. Also, potential failure modes were quantified and classified according to priority-risk number (Figure 1).

Following the classification of the risks, in the initial phase of the analysis of the causes of failure, special attention must be paid to the risks that exceed the acceptable level.

For design activities with medium or high risk, actions are needed to prevent the occurrence of these failure modes. All these actions have the role of reducing the risk of errors in design that could lead to system failures (Figure 2).

It can be observed that after applying the corrective actions, risks R6, R7, R8 and R9 are located in the area of medium risks. The distribution of potential risks by risk areas according to the priority-risk number of the optimized stage of the design compared to the initial stage indicates that all the analysed characteristics have an acceptable level, the risks being located in the medium and low risk areas, which means an improvement in the design process (Figure 2). 
RECENT, Vol. 20, no. 3(59), 2019

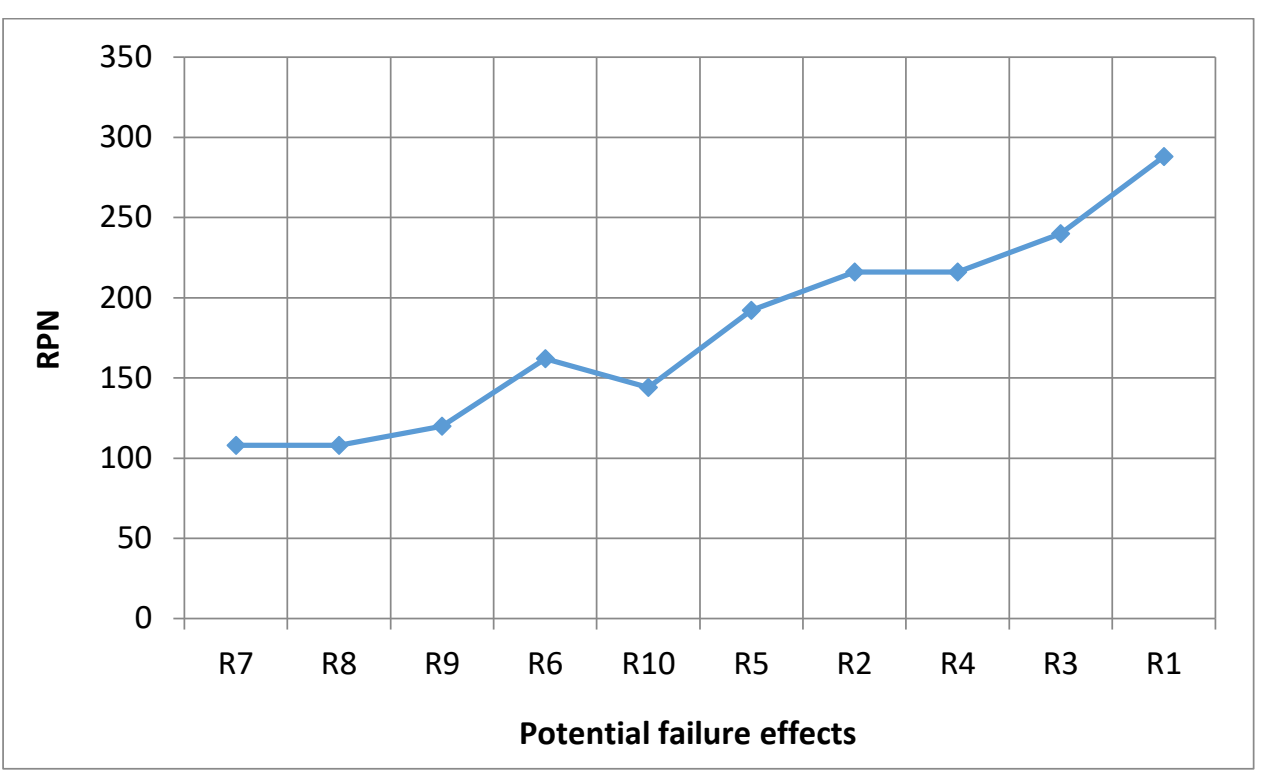

Fig. 1. Risk distribution for initial stage

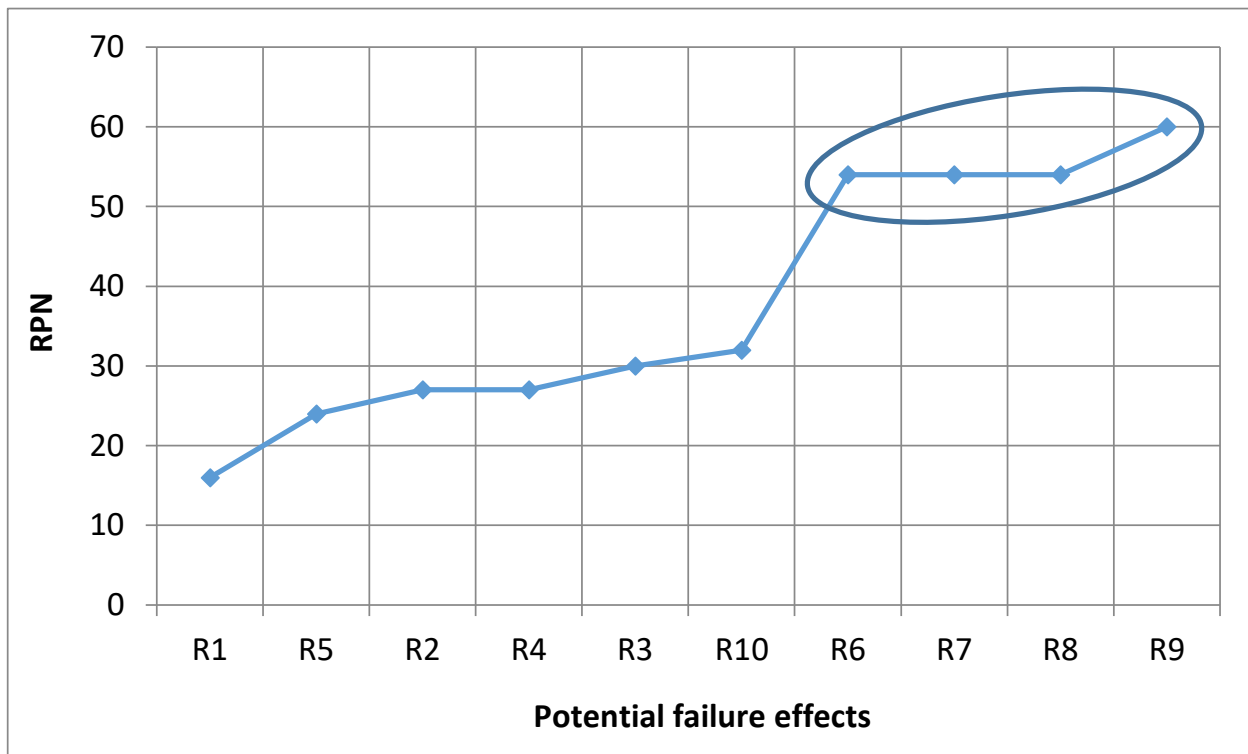

Fig. 2. Risk distribution for optimized stage

\section{Conclusions}

In the context of the design process, a variety of potential failure modes may appear, such as:

- impossibility of system assembly in the internal assembly process;

- the impossibility of assembling the system to the client;

- failure to perform the passenger restraint function;

- holding the passenger up to a certain force that is under the legal regulations.

In the event of an accident, the passenger's detention may become uncertain if:

- ECE R14 legal anchorage points are not respected;

- testing does not really represent the environment inside the car, the loading angle of the force is

different, the seat is not the same with the original one from the car and the deformation of the car

is not properly simulated in dynamic testing;

- it is not permitted to align the locking system strap with the force direction at the moment of impact;

- the wrong size of the fixing hole: it involves tolerances that have not been provided, so the locking system strap does not resist the applied forces resulting from the test;

- low tensile strength: it may result from interference with other elements of the car which lead to a 
decrease in the performance of the locking system;

- the critical section of the plate is not respected, thus causes breaking in case of an impact;

- the failure mode of the locking system strap is not the one followed in the test, so the occupant may be injured;

- sharp edges of the locking system strap may injure the passenger;

- locking system strap induces shocks to the locking head while traveling, which can lead to false locking of the belt buckle in the locking head and the passenger being not restrained in the event of an impact.

DFMEA is the optimum option for any organization because it can lead to lower non-quality costs and improved products quality.

The advantages of DFMEA implementation include:

- improving the quality, reliability and safety of a product or process;

- improving the image and competitiveness of the company;

- increasing consumer satisfaction;

- reduction of research and development times and costs;

- collecting information to reduce future failures and defects;

- reduction of potential warranty issues;

- early identification and elimination of potential defects;

- emphasizing the prevention of problems;

- minimizing the late changes that could be made to the product or process, as well as the related costs, etc.

\section{References}

1. Xiao N., Huang H.Z., Li Y., He L., Jin T. (2011): Multiple failure modes analysis and weighted risk priority number evaluation in FMEA. Engineering Failure Analysis, ISSN 1350-6307, Vol. 18, is. 4, p. 1162-1170, https://doi.org/10.1016/i.engfailanal.2011.02.004

2. Stamatis D.H. (2003): Failure mode and effect analysis: FMEA from theory to execution. American Society for Quality, Quality press, ISBN 0-87389-598-3

3. Pattnaik S. (2015): Design failure modes and effects analysis (DFMEA) of an all-terrain vehicle. International Journal of Research in Engineering and Technology, eISSN 2319-1163, Vol. 4, is. 6, p. 339-345, https://ijret.org/volumes/2015v04/i06/IJRET20150406058.pdf

4. Segismundo A., Augusto Cauchick Miguel P. (2008): Failure mode and effects analysis (FMEA) in the context of risk management in new product development: A case study in an automotive company. International Journal of Quality \& Reliability Management, ISSN 0265-671X, Vol. 25, No. 9, p. 899-912, https://doi.org/10.1108/02656710810908061

5. Spath P.L. (2003): Using failure mode and effects analysis to improve patient safety. AORN Journal, ISSN 18780369, Vol. 78, is. 1, p. 15-37, https://doi.org/10.1016/S0001-2092(06)61343-4

6. General Motors Corp. (2008): Potential Failure Mode and Effects Analyses (FMEA). Reference manual. Fourth edition. ISBN 978-1-60534-136-1

7. Rana S., Belokar R.M. (2017): Quality improvement using FMEA: A Short Review. International Journal of Research in Engineering and Technology (IRJET), eISSN 2319-1163, Vol. 4, is. 6, p. 263-267, https://www.irjet.net/ archives/V4/i6/IRJET-V4I645.pdf

8. Haq M.I., Anand A., Nasir T., Singh D. (2013): Design considerations for safety in automobiles: a system approach. International Journal of Mechanical Engineering and Research, ISSN 2249-0019, Vol. 3, No. 6, p. 583-588

9. McClafferty K., German A. (2008): High-tech motor vehicle safety systems. Proceedings of the $18^{\text {th }}$ Canadian Multidisciplinary Road Safety Conference, p. 1-17, https://roadsafetyresearch.ca/papers/cmrsc18 hightech.pdf

10. Bosch R. (2018): Bosch automotive handbook. 10th Edition. SAE International, ISBN 978-0768095678

11. Wang X.-Y., Jiang Y.-H., Li R.-B., Chen M.-Z. (2018): Automobile safety technology and its improvement. MATEC Web of Conferences 160:05012, https://doi.org/10.1051/matecconf/201816005012 